\title{
AN INDIVIDUAL ERGODIC THEOREM
}

\author{
RYOTARO SATO
}

Abstract. An individual ergodic theorem is proved for a linear operator $T$ on $L_{1}$ of a finite measure space which satisfies certain norm conditions.

Derriennic and Lin [5] showed by an example that given an $\varepsilon>0$ there exists a positive linear operator $T$ on $L_{1}$ of a finite measure space, with $T 1=1$ and $\left\|T^{n}\right\|_{1}=1+\varepsilon$ for all $n \geqslant 1$, and a function $f$ in $L_{1}$ such that the individual ergodic limit

$$
\lim _{n} \frac{1}{n} \sum_{i=0}^{n-1} T^{i} f(x)
$$

does not exist almost everywhere on a certain measurable subset of positive measure.

In this paper, however, we shall prove the following individual ergodic theorem.

TheOREM. Let $(X, \mathcal{F}, \mu)$ be a finite measure space and $L_{p}=L_{p}(X, \mathcal{F}, \mu)$, $1<p<\infty$, the usual Banach spaces. Let $T$ be a bounded linear operator on $L_{1}$ and $\tau$ its linear modulus in the sense of Chacon and Krengel [4]. Assume the conditions:

$$
\begin{aligned}
& \sup _{n}\left\|\frac{1}{n} \sum_{i=0}^{n-1} \tau^{i}\right\|_{1}<\infty, \\
& \sup _{n}\left\|\frac{1}{n} \sum_{i=0}^{n-1} \tau^{i}\right\|_{\infty}<\infty .
\end{aligned}
$$

Then, for any $f \in L_{\infty}$, the ergodic limit

$$
\lim _{n} \frac{1}{n} \sum_{i=0}^{n-1} T^{i} f(x)
$$

exists for almost all $x \in X$.

Proof. Let $T^{*}$ and $\tau^{*}$ denote the adjoint operators of $T$ and $\tau$, respectively. Since $\left|T^{*} f\right|<\tau^{*}|f|$ (cf. [1]) and $\int \tau^{*}|f| d \mu=\int(\tau 1)|f| d \mu \leqslant\|\tau\|_{\infty}\|f\|_{1}$ for all

Received by the editors November 17, 1976 and, in revised form, January 31, 1977. AMS (MOS) subject classifications (1970). Primary 47A35; Secondary 28A65.

Key words and phrases. Individual and mean ergodic theorems, Chacon's general ratio ergodic theorem, finite measure space, linear operator, linear modulus of a linear operator, norm conditions.

C American Mathematical Society 1977 
$f \in L_{\infty}, T^{*}$ and $\tau^{*}$ can be extended to bounded linear operators $S$ and $\sigma$ on $L_{1}$, respectively. It is easily seen that

$$
\left.S^{*}=T \quad\left(\text { on } L_{\infty}\right) \quad \text { and } \quad \sigma^{*}=\tau \quad \text { (on } L_{\infty}\right) .
$$

Hence it follows that the linear modulus of $S$ is $\sigma$ and that

$$
\sup _{n}\left\|\frac{1}{n} \sum_{i=0}^{n-1} \sigma^{i}\right\|_{1}=\sup _{n}\left\|\frac{1}{n} \sum_{i=0}^{n-1} \tau^{i}\right\|_{\infty}<\infty .
$$

We now define two functions $u$ and $v$ in $L_{\infty}$ by the relations:

$$
u(x)=\limsup _{n} \frac{1}{n} \sum_{i=0}^{n-1} \tau^{* i} 1(x) \quad(x \in X)
$$

and

$$
v(x)=\limsup _{n} \frac{1}{n} \sum_{i=0}^{n-1} \tau^{i} 1(x) \quad(x \in X) .
$$

It may be readily seen that $\tau^{*} u>u$ and $\tau v \geqslant v$. Thus if we set

and

$$
s(x)=\lim _{n} \frac{1}{n} \sum_{i=0}^{n-1} \tau^{* i} u(x) \quad(x \in X)
$$

$$
t(x)=\lim _{n} \frac{1}{n} \sum_{i=0}^{n-1} \tau^{i} v(x) \quad(x \in X),
$$

then

$$
\tau^{*} s=s \text { and } \tau t=t \text {. }
$$

Let $Y=\{x: s(x)>0\}$ and $Z=X-Y$. Then, by [5] and [7], we have:

(i) $f \in L_{1}(Z)$ implies $\tau f \in L_{1}(Z)$,

(ii) if $f \in L_{1}(Z)$ then $\lim _{n}\left\|(1 / n) \sum_{i=0}^{n-1} \tau^{i} f\right\|_{1}=0$,

(iii) $\lim _{n}\left\|\tau^{n} f\right\|_{1} / n=0$ for every $f \in L_{1}$.

We shall now divide the proof of the theorem into several steps, since it is rather long.

Step I. If $f \in L_{\infty}(Z)$ then $\lim _{n}(1 / n) \sum_{i=0}^{n-1} T^{i} f(x)=0$ for almost all $x \in X$.

To prove this, we may and do assume without loss of generality that $0<f<1$ on $Z$, and it is enough to show that the function $h$ defined by

$$
h(x)=\lim \sup _{n} \frac{1}{n} \sum_{i=0}^{n-1} \tau^{i} f(x) \quad(x \in X)
$$

satisfies $h(x)=0$ almost everywhere on $X$. In fact, we notice that $0 \leqslant h \in$ $L_{\infty}(Z)\left(\subset L_{1}(Z)\right)$, by (2) and (i), and that $\tau h \geqslant h$. Hence (ii) implies that

$$
\|h\|_{1}<\lim _{n}\left\|\frac{1}{n} \sum_{i=0}^{n-1} \tau^{i} h\right\|_{1}=0,
$$

and therefore $h(x)=0$ almost everywhere on $X$.

Step II. For any $f \in L_{\infty}$ the limit $\lim _{n}(1 / n) \sum_{i=0}^{n-1} T^{i} f(x)$ exists for almost all $x \in Y$. 
To see this, we first notice that

$$
\int|T f| s d \mu \leqslant \int(\tau|f|) s d \mu=\int|f| \tau^{*} s d \mu=\int|f| s d \mu
$$

for all $f \in L_{1}$. Thus $T$ may be regarded as a linear contraction operator on $L_{1}(Y, s d \mu)$, since $L_{1}=L_{1}(X, \mathcal{F}, \mu)$ is a dense subspace of $L_{1}(Y, s d \mu)$. Therefore we can apply Chacon's general ratio ergodic theorem [2], [3] to $T$ to infer that, for any $f \in L_{\infty}=L_{\infty}(X, \mathcal{F}, \mu)\left(\subset L_{1}(Y, s d \mu)\right)$, the limit

$$
\lim _{n} \frac{1}{n} \sum_{i=0}^{n-1} T^{i} f(x)=\lim _{n} t(x) \sum_{i=0}^{n-1} T^{i} f(x) / \sum_{i=0}^{n-1} \tau^{i} t(x)
$$

exists almost everywhere on $Y \cap\{x: t(x)>0\}$. On the other hand it is immediate from the definition of $t$ that, for any $f \in L_{\infty}$,

$$
\lim _{n} \frac{1}{n} \sum_{i=0}^{n-1} T^{i} f(x)=0
$$

almost everywhere on $\{x: t(x)=0\}$. Thus Step II is established.

Step III. If $f \in L_{\infty}$ and $\lim _{n}\left\|(1 / n) \sum_{i=0}^{n-1} T^{i} f\right\|_{1}=0$, then $\lim _{n}(1 / n) \sum_{i=0}^{n-1} T^{i} f(x)=0$ for almost all $x \in X$.

To prove this, define

$$
f_{0}(x)=\lim \sup _{n}\left|\frac{1}{n} \sum_{i=0}^{n-1} T^{i} f(x)\right| \quad(x \in X) .
$$

Clearly, $0<f_{0} \in L_{\infty}$, and it follows from Step II that $f_{0}(x)=0$ for almost all $x \in Y$. For each $k \geqslant 1$, put

$$
f_{k}=\frac{1}{k} \sum_{i=0}^{k-1} T^{i} f
$$

By an easy computation we then have

$$
\left|\frac{1}{n} \sum_{i=0}^{n-1} T^{i} f-\frac{1}{n} \sum_{i=0}^{n-1} T^{i} f_{k}\right|=\frac{1}{n}\left|\left(T^{n}-I\right) \sum_{i=1}^{k-1}\left(1-\frac{i}{k}\right) T^{i-1} f\right| \text {. }
$$

To see that

$$
\lim _{n}\left|\frac{1}{n} \sum_{i=0}^{n-1} T^{i} f(x)-\frac{1}{n} \sum_{i=0}^{n-1} T^{i} f_{k}(x)\right|=0
$$

for almost all $x \in X$, define

$$
h(x)=\left|\sum_{i=1}^{k-1}\left(1-\frac{i}{k}\right) T^{i-1} f(x)\right| \quad(x \in X)
$$

and

$$
\tilde{h}(x)=\lim \sup _{n} \frac{1}{n} \tau^{n} h(x) \quad(x \in X) .
$$

Then we have 


$$
\underset{n}{\limsup } \frac{1}{n}\left|T^{n}\left(\sum_{i=1}^{k-1}(1-i / k) T^{i-1} f\right)(x)\right|<\tilde{h}(x)
$$

for almost all $x \in X$, and furthermore

$$
\tau \tilde{h}(x)=\tau\left(\limsup _{n} \frac{1}{n} \tau^{n} h(x)\right) \geqslant \lim \sup _{n} \frac{1}{n} \tau^{n+1} h(x)=\tilde{h}(x)
$$

for almost all $x \in X$. Here we can apply Step II to $\tau$ instead of $T$ and obtain that $\tilde{h}(x)=0$ for almost all $x \in Y$. Therefore $\tilde{h} \in L_{\infty}(Z)\left(\subset L_{1}(Z)\right)$, and so

$$
\|\tilde{h}\|_{1}<\lim _{n}\left\|\frac{1}{n} \sum_{i=0}^{n-1} \tau i \tilde{h}\right\|_{1}=0
$$

by (ii). Consequently, $\tilde{h}(x)=0$ for almost all $x \in X$.

It then follows that, for almost all $x \in X$,

$$
\underset{n}{\lim \sup }\left|\frac{1}{n} \sum_{i=0}^{n-1} T^{i} f(x)-\frac{1}{n} \sum_{i=0}^{n-1} T^{i} f_{k}(x)\right|=0 \quad(k>1) .
$$

Thus, for almost all $x \in X$,

$$
\begin{aligned}
f_{0}(x) & =\limsup _{n}\left|\frac{1}{n} \sum_{i=0}^{n-1} T^{i} f_{k}(x)\right| \leqslant \limsup \frac{1}{n} \sum_{i=0}^{n-1} \tau^{i}\left|f_{k}\right|(x) \\
& <\limsup _{n} \frac{1}{n} \sum_{i=0}^{n-1} \tau^{i} f_{k}^{1}(x)+\limsup _{n} \frac{1}{n} \sum_{i=0}^{n-1} \tau^{i} f_{k}^{2}(x),
\end{aligned}
$$

where $f_{k}^{1}=\left|f_{k}\right| 1_{Z}$ and $f_{k}^{2}=\left|f_{k}\right| 1_{Y}$. By this and the argument in Step I, we obtain that

$$
f_{0}(x)<\lim \sup _{n} \frac{1}{n} \sum_{i=0}^{n-1} \tau^{i} f_{k}^{2}(x)
$$

for almost all $x \in X$, because $f_{k}^{1} \in L_{\infty}(Z)$.

Let us put, for each $k>1$,

$$
\tilde{f}_{k}(x)=\limsup _{n} \frac{1}{n} \sum_{i=0}^{n-1} \tau^{i} f_{k}^{2}(x) \quad(x \in X) .
$$

It follows that $0<\tilde{f}_{k} \in L_{\infty}$ and that $\tau \tilde{f}_{k}>\tilde{f}_{k}$. Hence, writing $g_{k}^{1}=\tilde{f}_{k} 1_{z}$ and $g_{k}^{2}=\tilde{f}_{k} 1_{Y}$, and applying (ii) to $g_{k}^{1} \in L_{\infty}(Z)\left(\subset L_{1}(Z)\right)$, we get

$$
\begin{aligned}
\left\|\tilde{f}_{k}\right\|_{1} & <\lim _{n}\left\|\frac{1}{n} \sum_{i=0}^{n-1} \tau^{i} \tilde{f}_{k}\right\|_{1} \\
& <\lim _{n}\left\|\frac{1}{n} \sum_{i=0}^{n-1} \tau^{i} g_{k}^{1}\right\|_{1}+\lim _{n}\left\|\frac{1}{n} \sum_{i=0}^{n-1} \tau^{i} g_{k}^{2}\right\|_{1} \\
& \left.=\lim _{n}\left\|\frac{1}{n} \sum_{i=0}^{n-1} \tau^{i} g_{k}^{2}\right\|_{1}<\sup _{n}\left\|\frac{1}{n} \sum_{i=0}^{n-1} \tau^{i}\right\|_{1}\right]\left\|g_{k}^{2}\right\|_{1} .
\end{aligned}
$$


On the other hand, applying Step II to $\tau$ instead of $T$, we observe that

$$
g_{k}^{2}(x)=\lim _{n}\left(\frac{1}{n} \sum_{i=0}^{n-1} \tau^{i} f_{k}^{2}(x)\right) 1_{Y}(x)
$$

for almost all $x \in X$. Hence, by Fatou's lemma,

$$
\begin{aligned}
\left\|g_{k}^{2}\right\|_{1} & \leqslant \lim _{n} \inf \left\|\left(\frac{1}{n} \sum_{i=0}^{n-1} \tau^{i} f_{k}^{2}\right) 1_{Y}\right\|_{1} \\
& \leqslant\left(\sup _{n}\left\|\frac{1}{n} \sum_{i=0}^{n-1} \tau^{i}\right\|_{1}\right)\left\|f_{k}^{2}\right\|_{1} .
\end{aligned}
$$

By this and the fact that

$$
\lim _{k}\left\|f_{k}^{2}\right\|_{1}=\lim _{k}\left\|\left(\frac{1}{k} \sum_{i=0}^{k-1} T^{i f}\right) 1_{Y}\right\|_{1}=0,
$$

we obtain that $\lim _{k}\left\|g_{k}^{2}\right\|_{1}=0$, and hence that $\lim _{k}\left\|\tilde{f}_{k}\right\|_{1}=0$. Since $0<f_{0}(x)$ $<\tilde{f}_{k}(x)$ for almost all $x \in X$, it must follow that $\left\|f_{0}\right\|_{1}=0$. So $f_{0}(x)=0$ almost everywhere on $X$, and this establishes Step III.

Step IV. For any $f \in L_{\infty}$ there exists a function $g \in L_{\infty}$ such that $\mathbf{T g}=g$ and $\lim _{n}\left\|(1 / n) \sum_{i=0}^{n-1} T^{i}(f-g)\right\|_{1}=0$.

To prove this, let $f \in L_{\infty}$ be given. Then, by (2) and Theorem IV.8.9 in [6], the set $\left\{(1 / n) \sum_{i=0}^{n-1} T^{i} f: n \geqslant 1\right\}$ is weakly sequentially compact in $L_{1}$, and by (iii), $\lim _{n}\left\|T^{n} f\right\|_{1} / n \leqslant \lim _{n}\left\|\tau^{n}|f|\right\|_{1} / n=0$. Hence, a well-known mean ergodic theorem (cf. Theorem VIII.5.1 in [6]) shows that there exists a function $g \in L_{1}$ such that $T g=g$ and

$$
\lim _{n}\left\|\frac{1}{n} \sum_{i=0}^{n-1} T^{i} f-g\right\|_{1}=0 .
$$

Clearly $g \in L_{\infty}$, by condition (2), and hence Step IV is established.

Step V. We shall now conclude the proof of the theorem as follows.

Let $f \in L_{\infty}$ be given, and using Step IV, write $f=g+h$ where $T g=g \in$ $L_{\infty}$ and $h\left(\in L_{\infty}\right)$ satisfies $\lim _{n}\left\|(1 / n) \sum_{i=0}^{n-1} T i h\right\|_{1}=0$. Then, by Step III, we observe that

$$
\lim _{n} \frac{1}{n} \sum_{i=0}^{n-1} T^{i} f(x)=g(x)
$$

for almost all $x \in X$. Hence the proof is completed.

REMARK. In [8], an analogous result is proved for a strongly continuous one-parameter semigroup $\left\{T_{t}\right\}_{0<t<\infty}$ of positive linear operators on $L_{1}$ of a finite measure space.

EXAMPLE. We shall construct a positive linear operator $T$ on $L_{1}$ of a finite measure space which satisfies the following norm conditions: 


$$
\begin{aligned}
& \sup _{n}\left\|\frac{1}{n} \sum_{i=0}^{n-1} T^{i}\right\|_{1}<\infty \text { and } \sup _{n}\left\|T^{n}\right\|_{1}=\infty, \\
& \sup _{n}\left\|\frac{1}{n} \sum_{i=0}^{n-1} T^{i}\right\|_{\infty}<\infty \text { and } \sup _{n}\left\|T^{n}\right\|_{\infty}=\infty .
\end{aligned}
$$

Put $a_{0}=0, a_{1}=1, a_{2}=2, a_{n}=4 a_{n-1}(n \geqslant 3)$, and $b_{n}=\sum_{i=0}^{n} a_{i}(n \geqslant 0)$. Write $X=\left\{(n, i): n \geqslant 1\right.$ and $\left.1 \leqslant i \leqslant b_{n}\right\}$, and let $\mathcal{F}$ be all possible subsets of $X$ and $\lambda$ the measure on $(X, \mathscr{F})$ defined by $\lambda(\{(1,1)\})=1$ and, for $n \geqslant 2$,

$$
\lambda(\{(n, i)\})= \begin{cases}1 / 2^{n-1}, & \text { if } 1 \leqslant i \leqslant a_{n}, \\ \lambda\left(\left\{\left(n-1, i-a_{n}\right)\right\}\right), & \text { if } a_{n}<i \leqslant b_{n} .\end{cases}
$$

Put $\mu(\{(n, i)\})=\left(2 / 4^{n}\right) \lambda(\{(n, i)\})$. Then it follows from an easy computation that $(X, \mathcal{F}, \mu)$ is a probability measure space. Define a point mapping $\varphi$ from $X$ to $X$ by the relation:

$$
\varphi((n, i))= \begin{cases}(n, i+1), & \text { if } 1 \leqslant i<b_{n}, \\ (n+1,1), & \text { if } i=b_{n} .\end{cases}
$$

Then $X=\left\{\varphi^{n}((1,1)): n \geqslant\right\}$, and thus if we set, for convenience's sake, $\varphi^{n}((1,1))=n$, then $X=\{n: n \geqslant 0\}$. Define a positive linear operator $S$ on $L_{1}(X, \mathcal{F}, \mu)$ by the relation:

$$
S f(n)=f(n+1) \quad\left(f \in L_{1}(X, \mathcal{F}, \mu), n \in X\right) .
$$

Then we have $\sup _{n}\left\|(1 / n) \sum_{i=0}^{n-1} S^{i}\right\|_{1} \leqslant 4$, since

$$
\frac{\mu\left(\left\{(n, i): 1 \leqslant i \leqslant b_{n}\right\}\right)}{b_{n} \mu(\{(n, 1)\})}<2 \quad(n \geqslant 1) .
$$

It is clear that $\sup _{n}\left\|S^{n}\right\|_{1}=\infty$.

Next, let $h$ be the function in $L_{\infty}(X, \mathcal{F}, \mu)$ defined by $h(n)=2^{-i+1}$, where $b_{i-1}<n+1<b_{i}$. Since

$$
\left(\sum_{i=0}^{b_{n}} h(i)\right) /\left(b_{n}+1\right) h\left(b_{n}\right)<4 \quad(n \geq 0),
$$

it follows that

$$
\sup _{n}\left\|\left(\frac{1}{n} \sum_{i=0}^{n-1} S^{i} h\right) / h\right\|_{\infty} \leqslant 4
$$

and also that $\sup _{n}\left\|\left(S^{n} h\right) / h\right\|_{\infty}=\infty$. Hence, if we define a positive linear operator $T$ on $L_{1}(X, F, h d \mu)$ by the relation:

$$
T f=S(f h) / h \quad\left(f \in L_{1}(X, \mathscr{F}, h d \mu)\right),
$$

then it is easily seen that $T$ satisfies norm conditions (7) and (8).

ACKNOWLEDGEMENT. In conclusion, the author would like to express his gratitude to the referee for helpful comments which improved the paper. 


\section{BIBLIOGRAPHY}

1. M. A. Akcoglu and A. Brunel, Contractions on $L_{1}$-spaces, Trans. Amer. Math. Soc. 155 (1971), 315-325.

2. R. V. Chacon, Operator averages, Bull. Amer. Math. Soc. 68 (1962), 351-353.

3. Convergence of operator averages, Ergodic Theory (Proc. Internat. Sympos., Tulane Univ., New Orleans, La., 1961), Academic Press, New York, 1963, pp. 89-120.

4. R. V. Chacon and U. Krengel, Linear modulus of a linear operator, Proc. Amer. Math. Soc. 15 (1964), 553-559.

5. Y. Derriennic and M. Lin, On invariant measures and ergodic theorems for positive operators, J. Functional Anal. 13 (1973), 252-267.

6. N. Dunford and J. T. Schwartz, Linear operators. I: General theory, Pure and Appl. Math., vol. 7, Interscience, New York, 1958.

7. R. Sato, Ergodic properties of bounded $L_{1}$-operators, Proc. Amer. Math. Soc. 39 (1973), $540-546$.

8. Ergodic theorems for semigroups of positive operators, J. Math. Soc. Japan (to appear).

Department of Mathematics, Josai University, SAKado, SATtama, 350-02 Japan 